\title{
Does routine screening for breast cancer raise anxiety? Results from a three wave prospective study in England
}

\author{
Stephen Sutton, Guitta Saidi, Graham Bickler, Janet Hunter
}

\begin{abstract}
Objective - To investigate whether mammography raises anxiety in routinely screened women who receive a negative result.

Design - Prospective design in which women completed questionnaires at three key points in the breast screening process: at baseline (before being sent their invitation for breast screening), at the screening clinic immediately before or after screening, and at follow up, about nine months after baseline. Information was obtained from non-attenders as well as from attenders.
\end{abstract}

Setting - Bromley District Health Authority, served by the South East London Breast Screening Service.

Participants - Two overlapping samples were used. Sample A comprised 1500 women aged 50-64 who were due to be called for first round screening at a mobile screening unit. Altogether 1021 (68\%) returned a usable questionnaire and 795 of these (78\%) also provided adequate information at nine month follow up: there were 695 attenders (including 24 women who received false positive results) and 100 non-attenders. Sample $B$ consisted of 868 women who attended the screening unit in a three month period, $732(84 \%)$ of whom provided adequate data. A total of 306 attenders (including 10 who received false positive results) occurred in both samples and provided adequate information on all occasions. The main analyses were based on these 306 women plus the 100 nonattenders. The analysis of retrospective anxiety took advantage of the larger sample size of 695 attenders.

ICRF Health Institute of Psychiatry, 4 Windsor Walk, London SE5 8AF $S$ Sutton

G Saidi

South East London Health Authority, 1 Lower Marsh, London SE1 7RJ

G Bickler

Bromley Health, Department of Public Health Medicine, Hayes, Kent BR2 7EH $\mathrm{J}$ Hunter

Correspondence to: Dr S Sutton.

Accepted for publication February 1995 screening process. They also reported having experienced more pain and discomfort during the $x$ ray.

Conclusions - Anxiety does not seem to be an important problem in routinely screened women who receive a negative result. This finding is very reassuring in relation to a major criticism of breast screening programmes. Thus, apart from maintaining current procedures such as keeping waiting times to a minimum, there seems to be no need to introduce special anxiety reducing interventions into the national programme. On the other hand, the findings for women who received false positive results suggest that there are aspects of the experience of being recalled for assessment after an abnormal mammogram that warrant further attention. The relationship between contemporaneous and retrospective anxiety should also be studied.

\section{(F Epidemiol Community Health 1995;49:413-418)}

There is increasing interest in assessing the potential psychological costs of screening and other medical procedures. ${ }^{1-4}$ It has been suggested that the anxiety aroused by breast screening may significantly offset the benefits of reduced mortality. ${ }^{5-7}$ Although critics of mass mammography emphasise the possible adverse psychological impact of receiving false positive results, it is important to establish whether routine screening for breast cancer also raises anxiety in the vast majority ( $>90 \%$ ) of women who receive a negative result. This was the main aim of the present study, which was conducted within the UK National Health Service Breast Screening Programme. A secondary aim of the study was to compare attenders and nonattenders with regard to anxiety in order to investigate the notion that screening programmes tend to attract women who are characteristically more (or less) anxious than average.

A number of studies have measured anxiety or psychiatric morbidity in women who receive a negative result after mammography..$^{8-11}$ These and other relevant studies ${ }^{12-14}$ have been reviewed elsewhere. ${ }^{4}$ Their results suggest that in general women who receive a negative result are not made anxious and may be reassured by their experience of participating in breast screening. None of the studies, however, included a baseline measure of anxiety, or more 


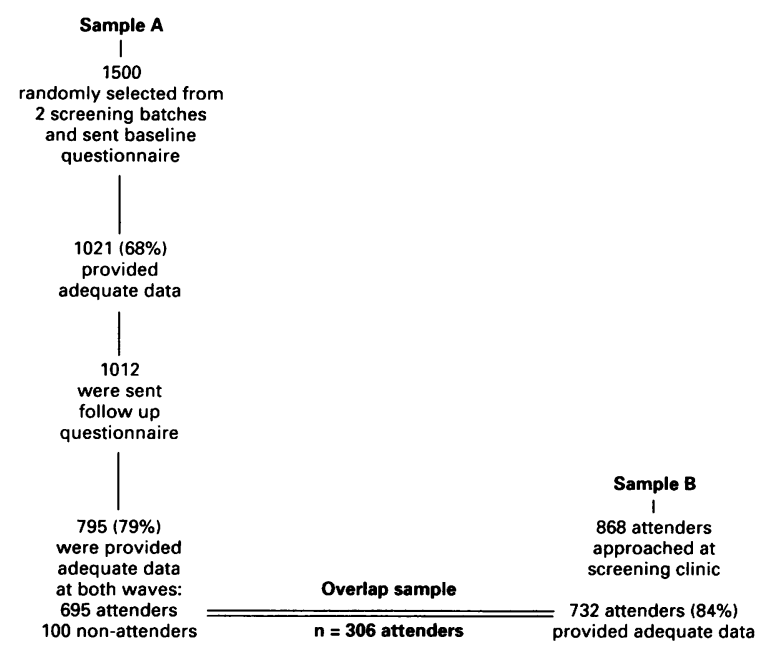

Fig 1 Flow diagram showing the design of the study.

than two waves of measurement, so it is difficult to exclude the possibility that attending for screening is associated with a real increase in anxiety which may persist for several months. Furthermore, few studies have assessed anxiety in those who fail to attend and thus have a direct bearing on the issue of whether breast screening programmes tend to attract women who are psychologically more (or less) healthy than average.

In the study reported here, a cohort of women was followed through the screening process, with anxiety measures at several key points at baseline (before invitation); at the screening clinic, either immediately before or immediately after screening; and at follow up, about nine months after baseline. Retrospective as well as contemporaneous measures of anxiety were used. Information was obtained from non-attenders as well as attenders. Although not the main focus of the present study, our sample included some women who were recalled for investigation after a positive breast screen but subsequently received a normal result. The results from this subgroup of subjects with "false positives" are also reported.

\section{Methods}

\section{SAMPLES}

Two overlapping samples, A and B, were used. Sample A consisted of 1500 women aged 50-64 living in Bromley District Health Authority who were due to be called for first round screening at the Bromley Mobile Breast Screening Unit. They were drawn randomly from two consecutive screening batches. Because of the way the screening batches were generated (by month and year of birth), the sample should be representative of women aged 50-64 who are registered with general practitioners in Bromley.

Sample B consisted of 868 women who attended the mobile unit for mammography between November 1991 and February 1992.

\section{DESIGN AND PROCEDURE}

One to three months before the invitations for screening were due to be sent out, women in sample A were sent a questionnaire through the post. A second questionnaire was sent to non-responders two to three weeks after the first. A follow up questionnaire was sent about nine months later, again with a second mailing to non-responders. Nine women who had been found to have breast cancer or were stil undergoing investigation were excluded from the follow up. However, 35 women ("false positives") who were recalled for investigation after a positive screen but were subsequently declared normal were included. Information on attendance was obtained from the screening office.

Sample A thus provided baseline (pre-invitation) and follow up measures of anxiety for both attenders and non-attenders. In order to obtain measures of anxiety from women (including those in sample A) while attending the screening clinic, four half-day clinic sessions per week were sampled at random over the three month period during which most women in sample $A$ would be expected to attend for screening. Women attending these sessions were approached by an experienced female interviewer (GS) and asked to complete a short questionnaire. Sessions were designated "before" or "after" sessions in a random fashion In "before" sessions, the questionnaires were administered while the women were waiting to be screened; in the "after" sessions, the questionnaires were administered immediately after they had been screened. Given the time and space constraints imposed by the environment of a mobile clinic, it would not have been practicable to obtain before and after measures from the same women. In addition, we wished to avoid creating a demand effect, women completing the questionnaire for the second time in the space of a few minutes may have felt subtle pressure to indicate that their anxiety had decreased. A total of 868 women were approached at the screening clinic (sample B). This procedure succeeded in its aim of producing a sizeable overlap between sample $A$ and sample $B$. The design of the study is shown in Figure 1.

\section{MEASURES}

Baseline questionnaire

The baseline postal questionnaire included the following measures:

(1) The Spielberger state-trait anxiety inventory (STAI, form-Y). ${ }^{15}$ This is a widely used, 40 item scale. The state scale was designed to assess transient states of anxiety (as might be experienced for example by a student about to sit an examination). In contrast, the trait scale is intended to assess a predisposition towards anxiety or the extent to which someone is characteristically anxious.

(2) The general health questionnaire (GHQ) anxiety subscale. The seven item anxiety subscale from the $\mathrm{GHQ}^{16}$ was also included.

(3) Sociodemographic factors. Items on age, marital status, education (whether respondent 
had any educational qualifications), employment (whether respondent did any paid work), housing tenure (an indicator of social class) were included.

\section{Clinic questionnaire}

Because of the time restriction, only the state anxiety questionnaire was used at the screening clinic.

\section{Follow up questionnaire}

The nine month follow up questionnaire repeated the anxiety scales from the baseline questionnaire. Among those who had attended for screening, retrospective ratings of anxiety, pain, and discomfort were also obtained.

\section{FINAL SAMPLES}

Sample $A$

Altogether 1021 out of 1500 women (68\%) returned a usable baseline questionnaire, and $795(78 \%)$ of these $(53 \%$ of 1500$)$ provided adequate data at baseline and follow up. There were 695 attenders (including 24 with false positive results) and 100 non-attenders.

\section{Sample B}

Of the 868 women approached at the screening clinic, $732(84 \%)$ provided adequate data. Common reasons for women refusing to fill out the questionnaire or failing to finish it were lack of time and not having their reading glasses with them.

\section{Overlap between $A$ and $B$}

A total of 306 attenders (including 10 women who received false positive results) occurred in both samples and provided adequate information at all stages. The main analyses of change in anxiety were based on these 306 attenders plus the 100 non-attenders from sample $A$ who provided adequate information at baseline and follow up. In this subsample of 406 women, the mean age was 58 years, $76 \%$ were married or living as married $(17 \%$ divorced, separated or widowed; $7 \%$ single), $50 \%$ had an educational qualification, $53 \%$ were in paid employment, and $91 \%$ owned or were buying their home. The subsample of 406 was very similar in both demographic characteristics and initial anxiety levels to the full sample of 1021 who returned a usable baseline questionnaire; thus there was no evidence of strong attrition bias. The analysis of retrospective anxiety (which was assessed on the follow up questionnaire) used a larger sample size of 695 attenders who provided usable information at this stage.

\section{STATISTICAL ANALYSIS}

The main procedures employed in the analysis were product-moment correlations, independent and paired $t$ tests, and repeated measures analysis of variance (ANOVA). Two sided significance tests and an alpha level of .05 were used throughout. The main analyses were repeated, controlling for each of the demographic factors in turn. Since the main results and conclusions were unchanged, only the original, unadjusted results are reported. Because the anxiety scores were highly skewed, the main results were checked - firstly, by using log transformed scores and secondly, by using nonparametric tests. All analyses were done using SPSSPC 4.1.

\section{Results}

COMPARISON OF STATE ANXIETY LEVELS

IMMEDIATELY BEFORE AND AFTER SCREENING

There was no significant difference between state anxiety levels immediately before and after screening. Some women, however, filled in the questionnaire after instead of before screening. This could have biased the before-after comparison if these women had had relatively high anxiety levels. To check on this, the analysis was repeated after excluding the 36 women for whom it had not been possible to adhere to the random allocation procedure. The results were unchanged; in particular, there was still no suggestion of a before-after difference in state anxiety. In subsequent analyses of state anxiety, the distinction between before and after screening is not retained; we simply use a single measure to represent the level of state anxiety experienced at the screening clinic.

\section{CHANGES IN ANXIETY LEVELS DURING THE}

SCREENING PROCESS

Mean anxiety scores at different stages in the screening process are shown in figure 2 for

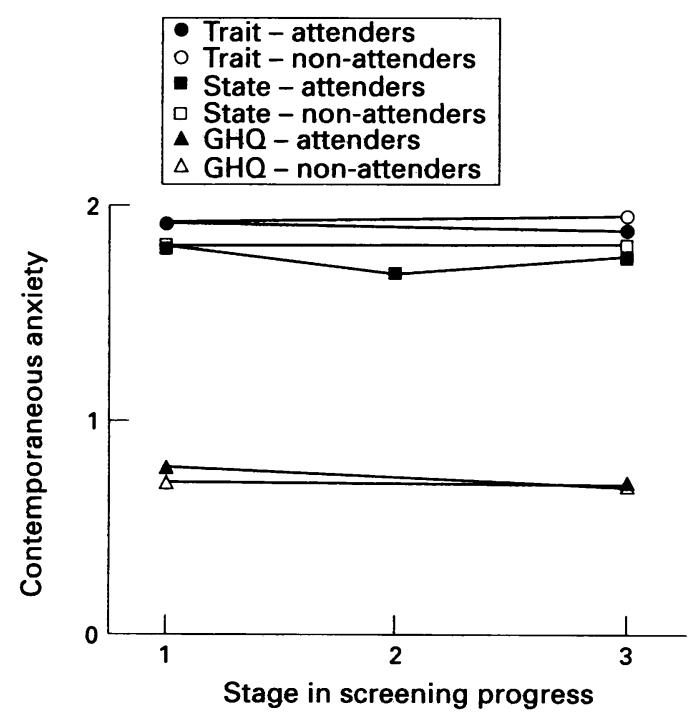

Figure 2 Mean anxiety scores for attenders and nonattenders at baseline (1), at clinic (2), and at follow up (3). All three anxiety questionnaires used four point scales and were scored conventionally as follows:

State anxiety: 1 = "not at all", 2= "somewhat", $3=$ State anxiety: $1=$ "not at all", $2=$ "moderately so", $4=$ "very much so".
"

Trait anxiety: 1= "almost never", 2 = "sometimes", $3=$ "often", 4 = "always".

GHQ anxiety: $0=$ "not at all", $1=$ "no more than usual", 2= "rather more than usual", 3= "much more than usual".

Note: Although it is common practice to report mean total scores on the state and trait anxiety scales (theoretical range 20-80), we report mean mean scores (theoretical range 1-4) because some participants had missing data on some items. 


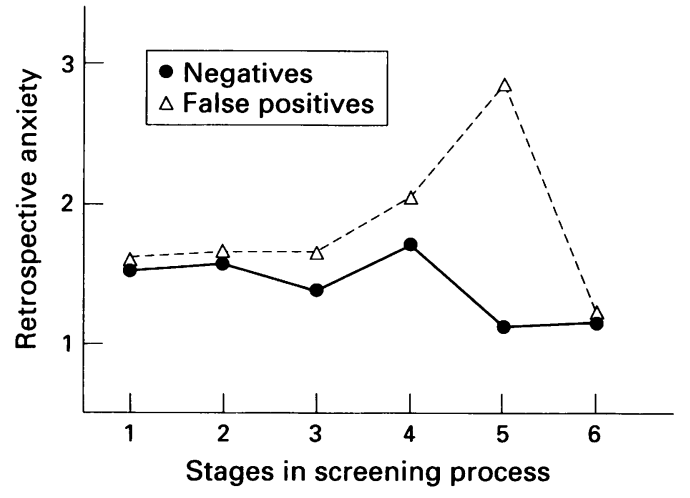

Figure 3 Mean retrospective anxiety for the two groups of attenders at each of six stages in the breast screening process. Key to stages:

$1=$ "when you received the invitation letter";

2 = "at the screening centre while you were waiting to have the $\mathrm{x}$ ray";

$3=$ "at the screening centre after you had had the $\mathrm{x}$ ray";

4'= "in the period between going for screening and receiving your results";

$5=$ "after you'd opened and read the results letter"; $6=$ "now".

attenders and non-attenders separately. The means for state and trait anxiety all fell within the range 1.65-1.95. Although somewhat higher than published norms for women in this age group, they are fairly typical of values obtained from working adults tested under unstressful conditions. ${ }^{15}$ (Psychiatric and general medical and surgical patients typically have mean scores $>2 \cdot 0$ ). State anxiety was consistently lower than trait anxiety, which suggests that the women were not unduly stressed. ${ }^{15}$ Repeated measures ANOVAs conducted on each of the three anxiety scales using the baseline and follow up data showed no significant differences between attenders and non-attenders (no main effect for group). Neither state nor trait anxiety showed a significant change from baseline to follow up. By contrast, GHQ anxiety showed a significant reduction over time: $\mathrm{F}(1,404)=11 \cdot 4, \mathrm{p}<\cdot 001$. The change was very small, however, in absolute terms (equivalent to less than one tenth of a unit on the four-point scale). None of the group (attenders $v$ non-attenders) analyses in relation to time interactions was significant. Thus there was no evidence that baseline anxiety predicted attendance and little evidence that attendance influenced anxiety, at least in the longer term.

Correlations between individuals' anxiety scores at baseline and follow up indicated considerable stability over time, particularly for trait anxiety $(r=0 \cdot 79, \mathrm{p}<\cdot 001)$.

Additional analyses incorporating demographic factors did not change the results reported above but showed a consistent effect of age. Even within the restricted age range studied here, the younger women were more anxious on each of the three anxiety scales. For example, the correlation between age and baseline trait anxiety score was $-0 \cdot 14(p<\cdot 01)$; the other correlations were of a similar order.

Among the attenders, state anxiety was assessed on three occasions (see fig 2 ). The three means differed significantly $(F(2,304)=7 \cdot 54$, $\mathrm{p}<\cdot 001)$. However, the expected pattern of change (an increase between baseline and clinic followed by a reduction at follow up) did not emerge. On the contrary, women were least anxious, on average, when they were at the screening clinic. The changes were again quite small in absolute terms.

Excluding the 10 women with false positive results did not change the results reported above. Repeated measures ANOVAs comparing the false positives with the other attenders (that is, the negative screenees) showed no differences between the two groups in anxiety levels or in the pattern of change in anxiety over time.

\section{ANALYSIS OF RETROSPECTIVE ANXIETY}

On the follow up questionnaire, those who had attended for screening were asked to rate how anxious they felt at each of six stages in the screening process. A simple three point scale was used $(1=$ not at all anxious, $2=\mathrm{a}$ bit anxious, 3 =very anxious). As figure 3 shows, retrospective anxiety varied considerably across stages and showed a different pattern in the two groups. (Repeated measures ANOVA comparing the false positives with the negative screenees yielded significant effects for group $(\mathrm{F}(1,665)=28 \cdot 6, \mathrm{p}<\cdot 001)$, for time $(\mathrm{F}(5$, $661)=143.4, p<\cdot 001)$, and for the group by time interaction $(\mathrm{F}(5,661)=63 \cdot 7, \mathrm{p}<\cdot 001)$.) As would be expected, compared with the negative screenees, the women with false positive results recalled feeling extremely anxious at stage 5 (after they had opened and read the results letter): mean (SD) $2 \cdot 85(\cdot 37)$ and 1.16 $(\cdot 36) ; \mathrm{t}(665)=21 \cdot 1, \mathrm{p}<\cdot 001$. What is of particular interest, however, is that retrospective anxiety was also higher in this group than in the negative screenees at stage 3 of the process, that is when they were at the screening clinic after being screened (mean (SD) $1.60(.68)$ and $1.36(\cdot 52)$ respectively; $\mathrm{t}(665)=2 \cdot 01, \mathrm{p}<\cdot 05)$. The difference in anxiety at stage 4 (in the period between going for screening and receiving their results) was also close to significance (mean (SD) $1.95(.09)$ and $1.70(.57)$ respectively; $\mathrm{t}(665)=1.93, \mathrm{p}=.054)$. The women with false positive results also reported having experienced significantly more pain and discomfort during the $x$ ray than women with negative results (mean (SD) for pain on a fourpoint scale from $1=$ no pain to $4=$ severe pain were $2.04(0.98)$ and $1.62(0.70)$ respectively; $t(680)=2 \cdot 80, p<\cdot 005)$. Thus, for this subgroup of women, the memory of the experience of going for screening seemed to have been coloured by their later experiences.

Retrospective anxiety at stage 6 ("now") correlated $\cdot 27-\cdot 28(\mathrm{p}<\cdot 001)$ with state, trait and GHQ anxiety as assessed on the follow up questionnaire.

\section{Discussion}

In this study we used both contemporaneous and retrospective measures to assess anxiety levels at key stages in the breast screening process. The former consisted of standardised 
multi-item scales whereas the latter were based on simple ad hoc ratings. The results for these two kinds of measures will be summarised and discussed in turn.

CONTEMPORANEOUS MEASURES OF ANXIETY Comparison of anxiety levels based on the contemporaneous measures with normative data indicated that the women were not especially anxious at any of the three points in the process. The expected pattern of change did not emerge. Among attenders, there was no difference between (state) anxiety levels immediately before and immediately after screening, and anxiety was at its lowest, on average, at the clinic, and highest at baseline. The changes were very small in absolute terms. One interpretation of the observed pattern of change is that the baseline questionnaire and covering letter prompted many women to think about the prospect of breast screening for the first time and thus produced a slight increase in anxiety. Thus, the initial measure of anxiety may not have been a true baseline. It may also have served to prepare the women for the forthcoming invitation for screening and preempted some of the anxiety that this communication might otherwise have aroused. There is also some evidence, at least for the GHQ, that a second administration of the instrument tends to yield lower scores than the first administration. ${ }^{18}$ If such an effect also occurs with the Spielberger scale, this may partly account for the slight reduction in state anxiety between baseline and clinic. Although the different waves of measurement took place at different times of year (baseline questionnaire in the autumn; clinic questionnaire in the winter; follow up questionnaire in the summer), seasonal effects seem an unlikely explanation for the particular pattern of results observed. It is unclear why anxiety was not higher while women were waiting to be screened and why no reduction was detectable from immediately before to immediately after screening. These findings may simply indicate that most women treat mammography as a routine test and take the experience in their stride.

\section{RETROSPECTIVE ANXIETY}

Among the women with negative results, retrospective anxiety (as assessed on the follow up questionnaire) stayed fairly constant over successive stages of the screening process until they had received the letter informing them of a negative result, at which point their anxiety was noticeably lower. This reduction was maintained at follow up (fig 3). These findings are different from the results based on the contemporaneous measures mainly in that retrospective anxiety was lowest at follow up. The women with false positive results, on the other hand, showed peak anxiety after they had read their results letter. The findings show an intriguing difference between the false positive and the negative screenees in the two stages before this. The false positive screenees had higher retrospective anxiety levels at the clinic after they had been screened and in the period between going for screening and receiving their results. They also recalled having experienced more pain and discomfort. The most plausible interpretation of this finding is that these women's memories of the earlier stages of screening were tainted by their later experiences. An alternative interpretation is that they really were more anxious at these stages in the screening process and really did experience more pain and discomfort. Our data enabled only a limited check on this possibility and the outcome was inconclusive. Gram and Slenker ${ }^{11}$ reported a similar finding which they interpreted in terms of possible physical differences between the false positives and the negative screenees: “... women in the FP (false positive) group have breasts that are more difficult to examine due to size or density thus necessitating a stronger and more painful compression of the breasts" ( $p$ 251). We are unaware of any evidence that bears directly on this suggestion, though Rutter $e a^{19}{ }^{19}$ found pain and discomfort during mammography to be unrelated to bust size and physical build. Whichever interpretation is preferred, it is likely to be retrospective anxiety rather than anxiety actually experienced that affects women's readiness to go for screening in the future. It should be reiterated that our study was not designed primarily to assess changes in anxiety among women who receive false positive results - a separate prospective study of this group is under way.

\section{COMPARISON OF ATTENDERS AND \\ NON-ATTENDERS}

We found no differences between attenders and non-attenders at baseline on any of the three (contemporaneous) anxiety scales. In other words, anxiety did not predict attendance. Thus, there is no evidence in our data that the NHS Breast Screening Programme is tending to attract women who are psychologically more or less healthy than average. This extends our knowledge of the factors that predict and fail to predict breast screening uptake, summarised recently by Calnan. ${ }^{20}$

\section{GENERALISABILITY OF THE FINDINGS}

The study was conducted in a district with an above average uptake of breast screening in women with high rates of home ownership. The results may not be generalisable to areas with lower uptake and to less advantaged women. The current research was done in a mobile clinic which might, if anything, be expected to produce higher anxiety levels at screening compared with the generally more pleasant and spacious static units. Because of the time restriction, only the state anxiety questionnaire was used at the screening clinic. It would have been preferable to use all three anxiety scales at all stages. Although the response rates at each wave were good, there was inevitably some attrition across waves (for example, only just over half the original sample 
of 1500 provided adequate information at baseline and follow up). While there was no evidence that this introduced significant bias, our findings may not be generalisable to women who do not return questionnaires. It is possible that those who neither returned the baseline questionnaire nor attended for screening were a particularly anxious group. Inevitably, as with previous studies, the results are limited by the measuring instruments used. There are many ways of measuring anxiety and related concepts like distress and psychiatric morbidity. It is not unusual in this field to find that different measures produce different results. The present study employed standardised anxiety scales that were not designed to be specific to breast cancer and breast screening. It is possible that more specific instruments, perhaps along the lines of that developed by Cockburn et al, ${ }^{21}$ would have yielded a different pattern of results.

\section{Conclusions}

Anxiety does not seem to be an important problem in routinely screened women who receive a negative result. This finding is very reassuring in relation to a major criticism of breast screening programmes. Thus, apart from maintaining commonsense strategies, such as giving women clear explanations of what breast screening involves and why it is being offered, and keeping waiting times to a minimum, ${ }^{22}$ there seems to be no need to introduce special anxiety reducing interventions into the national programme, at least as far as the negative screenees are concerned. Our results extend those of previous studies in suggesting that there are aspects of the experience of being recalled for assessment after an abnormal mammogram that warrant further attention. They also indicate the need for research on the relationship between contemporaneous and retrospective anxiety.

This research was funded by the Imperial Cancer Research Fund (ICRF). We thank the following for their contribution to the study: Dr Heather Nunnerley, Mrs Pam Bowell, and Mrs Linda Pollard of the South East London Breast Screening Service; Mrs Brenda Collier of Bromley FHSA; our colleagues draft of the paper; and all the women who participated.

1 Marteau TM. Psychological costs of screening. BMF 1989; 299:527.

2 Marteau TM. Reducing the psychological costs. BMF 1990; 301:26-8.

3 Wardle J, Pope R. The psychological costs of screening for cancer. $\mathcal{F}$ Psychosom Res 1992;36:609-24.

4 Sutton SR. Experiences of screening: anxiety issues. In: Austoker J, Patnick J, eds. Report of UKCCCR/NHSBSP workshop on breast screening acceptability: research and practice. Sheffield: NHSBSP, 1993. NHSBSP Publication no 28.

5 Roberts MM. Breast screening: time for a rethink? BMF 1989;299:1153-5.

6 Wright CJ. Breast cancer screening: a different look at the evidence. Surgery 1986;100:594-8.

7 Schmidt JG. The epidemiology of mass breast cancer screening - a plea for a valid measure of benefit. $\mathcal{J}$ Clin Epidemiol ing - a plea for a

8 Dean C, Roberts MM, French K, Robinson S. Psychiatric morbidity after screening for breast cancer. $\mathcal{f}$ Epidemiol Community Health 1986;40:71-5.

9 Ellman R, Angeli N, Christians A, Moss S, Chamberlain J, Maguire P. Psychiatric morbidity associated with screening for breast cancer. Br $\mathcal{F}$ Cancer 1989;60:781-4.

10 Bull AR, Campbell MJ. Assessment of the psychological impact of a breast screening programme. Br f Radiol 1991; 64:510-5.

11 Gram IT, Slenker SE. Cancer anxiety and attitudes toward mammography among screening attenders, nonattenders, and women never invited. Am $¥$ Public Health 1992;82: and wormer 241 .

12 Gram IT, Lund E, Slenker SE. Ouality of life following a false positive mammogram. Br f Cancer 1990;62:1018-22. false positive mammogram. Br f Cancer 1990;62:1018-22. 3 Lerman C, Trock B, Rimer BK, Boyce A, Jepson C, Eng-
strom PF. Psychological and behavioral implications of strom PF. Psychological and behavioral implications of
abnormal mammograms. Ann Intern Med 1991;114:657abnormal mammograms. Ann Intern Med 1991;114:657-

14 Lerman C, Trock B, Rimer BK, Jepson C, Brody D, Boyce A. Psychological side effects of breast cancer screening. Health Psychol 1991;10:259-67.

15 Spielberger CD. Manual for the state-trait anxiety inventory STAI-form Y. Palo Alto: Consulting Psychologists Press, 1983.

16 Goldberg DP, Williams P. A user's guide to the general health questionnaire. Windsor: NFER-Nelson, 1988.

17 Sutton SR, Bickler G, Sancho-Aldridge J, Saidi G. Prospective study of predictors of attendance for breast screening in inner London. $f$ Epidemiol Community Health 1994; 48:65-73.

18 Goldberg DP. Manual of the general health questionnaire. Windsor: NFER-Nelson, 1978 .

19 Rutter DR, Calnan M, Vaile MSB, Field S, Wade KA. Discomfort and pain during mammography: description, prediction, and prevention. $B M \mathcal{F}$ 1992;305:443-5.

20 Calnan M. Predictors of attendance. In: Austoker J, Patnick J, eds. Report of UKCCCR/NHSBSP workshop on breast screening acceptability: research and practice. Sheffield: NHSBSP, 1993. NHSBSP Publication no 28

21 Cockburn J, De Luise T, Hurley S, Clover K. Development and validation of the PCQ: a questionnaire to measure the psychological consequences of screening mammography. Soc Sci Med 1992;34:1129-34.

22 Saidi G. Off screen blues. Health Service fournal 1993;103(4 Nov): 26-7. 